\title{
Promising thermoelectric properties of phosphorenes
}

\author{
Cem Sevik ${ }^{1,3}$ and Hâldun Sevinçli ${ }^{2}$ \\ ${ }^{1}$ Department of Mechanical Engineering, Faculty of Engineering, Anadolu University, 26555, Eskişehir, \\ Turkey \\ ${ }^{2}$ Department of Materials Science and Engineering, Izmir Institute of Technology, Izmir, TR 35430, \\ Turkey \\ E-mail: csevik@anadolu.edu.tr and haldunsevincli@iyte.edu.tr
}

Received 4 March 2016, revised 17 June 2016

Accepted for publication 20 June 2016

Published 25 July 2016

\begin{abstract}
Electronic, phononic, and thermoelectric transport properties of single layer black- and bluephosphorene structures are investigated with first-principles based ballistic electron and phonon transport calculations employing hybrid functionals. The maximum values of room temperature thermoelectric figure of merit, $Z T$ corresponding to armchair and zigzag directions of blackphosphorene, $\sim 0.5$ and $\sim 0.25$, are calculated as rather smaller than those obtained with firstprinciples based semiclassical Boltzmann transport theory calculations. On the other hand, the maximum value of room temperature $Z T$ of blue-phosphorene is predicted to be substantially high and remarkable values as high as 2.5 are obtained for elevated temperatures. Besides the fact that these figures are obtained at the ballistic limit, our findings mark the strong possibility of high thermoelectric performance of blue-phosphorene in new generation thermoelectric applications.
\end{abstract}

Keywords: thermoelectricity, phosphorene, ballistic transport, thermal conductivity

(Some figures may appear in colour only in the online journal)

\section{Introduction}

Triggered by the realization of graphene, research on novel two-dimensional materials has become one of the major topics in materials science and condensed matter physics. New two-dimensional crystals have been investigated intensively [1] and several of them have been successfully fabricated, such as hexagonal boron nitride [2-4], silicene [5-8], and a large family of layered transition metal dichalcogenides [9-12]. Recently, a single layer of phosphorus, named as black-phosphorene (black-P) has been exfoliated $[13,14]$ and revealed as a new member of candidate materials for nanoelectronics applications [15-19] such as field-effect transistors [20, 21], wide-range photodetectors [22, 23], and high-performance lithium-ion batteries [24]. Following the experimental realization, other possible phosphorus based layered structures have been investigated and different stable allotropes have been determined by first-principles investigations

\footnotetext{
3 Author to whom any correspondence should be addressed.
}

[25-27]. Blue-phosphorene (blue-P) stands out among those as being the most stable structure, having only a few $\mathrm{meV}$ higher cohesive energy [27] than that of black-P.

Black-P was found to be a direct band-gap semiconductor with an estimated band gap of $\sim 2 \mathrm{eV}$ [16] and its carrier mobility has been predicted to be as high as $\sim 1000 \mathrm{~cm}^{2} \mathrm{~V}^{-1} \mathrm{~s}^{-1}$ [14]. In addition, as a result of first-principles based solution of the phonon Boltzmann transport equation, the room temperature lattice thermal conductivity, $\kappa_{\mathrm{L}}$, of this material has been estimated in the range of 30 to $110 \mathrm{Wm}^{-1} \mathrm{~K}^{-1}$ and 13 to $36 \mathrm{Wm}^{-1} \mathrm{~K}^{-1}$ along zigzag (ZZ) and armchair (AC) directions [28-33] respectively. Due to such superior electronic transport properties and relatively low $\kappa_{\mathrm{L}}$ (compared with the $\kappa$ of graphene, 2000 $-5000 \mathrm{Wm}^{-1} \mathrm{~K}^{-1}$ [34]), black-P has been suggested as a potential material for new generation nano thermoelectric applications. As a matter of fact, the room temperature thermoelectric figure of merit (ZT) of two-dimensional and quasione-dimensional (ribbon) black-P have been estimated to be as high as 1.0 [28] and 6.4 [35] respectively, using the 
semiclassical Boltzmann theory within the relaxation time approximation. In addition, a remarkable increase in the Seebeck coefficient and electrical conductivity of black-P with strain induced band convergence, which leads to a maximum room temperature $Z T$ value $\sim 2.1$ [36] have also been reported using the same method. However, as a result of first-principles calculations, Liao et al have revealed that the maximum room temperature $Z T$ value is only around $\sim 0.06$ [37]. On the other hand, the relatively high performance might be expected from blue-P and therefore it is worthwhile to shed light on its thermoelectric properties, as well. Besides these results on the thermoelectric performance of black-P, that of blue-P has not been investigated in detail yet.

We report ballistic transport calculations through which we investigate the performance limits of these materials and we compare and contrast the electronic, phononic, and thermoelectric transport properties of two types of single layer phosphorene structures, namely, black- and blue-phosphorenes. Our results for black-P are comparable with those previously reported by Liao et al [37]. On the other hand, quite efficient thermoelectric properties, even at moderate temperatures, are predicted for the blue-P structure, which suggest that blue-P is a strong candidate for future twodimensional thermoelectric applications.

\section{Method}

The electronic properties and the interatomic force constants are obtained using the Vienna $a b$ initio Simulation Package (VASP) $[38,39]$ which is based on density functional theory (DFT) [40-42]. The projector augmented wave pseudopotentials (PAW) [43, 44] from the standard distribution are incorporated in the calculations. For electronic exchangecorrelation functional, the generalized gradient approximation (GGA) in its Perdew-Burke-Ernzerhof (PBE) parameterization [45] is used. In order to capture the effect of exchange-correlation energy functionals particularly on electronic transport properties, we also use the screened Heyd -Scuseria-Ernzerhof (HSE06) hybrid functionals [46], which mix GGA with the exchange from Hartree-Fock theory [47]. The vibrational frequencies are obtained by using PHONOPY code [48], which can directly use the force constants calculated by density functional perturbation theory [49] as implemented in the VASP code. Here, $4 \times 3 \times 1$ $(28 \times 24 \times 1)$ and $5 \times 5 \times 1(30 \times 30 \times 1)$ conventional super cell structures ( $\Gamma$ centered k-points grids) are considered for black-P and blue-P respectively. A plane wave energy cut off and total energy convergence criterion of $500 \mathrm{eV}$ is used in all simulations, while the force tolerance is set to $10^{-2} \mathrm{eVA}^{-1}$. To prevent spurious interaction between isolated layers, a vacuum spacing of at least $15 \AA$ is introduced.

Vibrational thermal conductance is calculated from [50],

$$
\kappa_{\mathrm{ph}}=\int \frac{\mathrm{d} \omega}{2 \pi} \hbar \omega \frac{\partial f_{\mathrm{B}}(\omega, T)}{\partial T} \tau_{\mathrm{ph}}(\omega)
$$

where $\omega$ is the vibrational frequency, $f_{\mathrm{B}}$ stands for the Bose function, and $\tau_{\mathrm{ph}}$ is the phonon transmission spectrum. In the case of the electrons, we use the functions [51],

$$
L_{n}=-\frac{2}{h} \int \mathrm{d} E \frac{\partial f_{F}(E, \mu, T)}{\partial E}(E-\mu)^{n} \tau_{\mathrm{el}}(E)
$$

where $n$ is integer, $\mu$ is the chemical potential, $T$ is temperature, $f_{\mathrm{F}}$ is the Fermi function, and $\tau_{\mathrm{el}}$ is the electronic transmission spectrum. Using $L_{n}$, the electrical conductance, Seebeck coefficient, and the electrical contribution to thermal conductance are calculated as $G=e^{2} L_{0}, S=\left(L_{1} / L_{0}\right) / e T$ and $\kappa_{\mathrm{el}}=\left(L_{2}-L_{1}^{2} / L_{0}\right) / T$ respectively. Accordingly, the power factor and thermoelectric figure of merit are predicted using $P=S^{2} G$ and $Z T=S^{2} G T /\left(\kappa_{\mathrm{el}}+\kappa_{\mathrm{ph}}\right)$ respectively. In the ballistic limit, the transmission spectra can be obtained from the number of band crossings of a particular energy value. In order to obtain accurate results, $200 \times 200 k$-points for $\mathrm{PBE}$ and $100 \times 100 k$-points for HSE06 functionals are used to capture accurate electron transmission spectra. In addition, $100 q$-points are used in the transverse direction to capture accurate phonon transmission spectra.

\section{Results}

Figure 1 shows the structural representation of in plane and out of plane views of black-P and blue-P crystals represented by rectangular and hexagonal primitive unit cells with the space groups $D_{2 h}^{7}$ and $D_{3 h}^{3}$, respectively. These structures with a nonzero intrinsic thickness differ in the arrangements of tetrahedrally coordinated $\mathrm{P}$ atoms in $2 \mathrm{D}$ lattice. The optimized first-principles lattice parameters, $a_{1}=3.34 \AA$, and $a_{2}=4.62 \AA$ for black-P and $a_{1}=3.28 \AA$ for blue-P are in agreement with the previously reported values [21, 26, 27, 52].

The electronic properties of both materials are calculated using both PBE and HSE06 functionals as shown in figures 2(a) and (b). HSE06 functional results in a notable shift of the conduction bands, while the properties such as the group velocities and effective masses are not affected considerably. The determined HSE06 (PBE) band-gap values, 1.59 (0.91) eV for black-P and 2.73 (2.04) eV for blue-P are in quite good agreement with the previously reported values, 0.90 [26, 53] and $0.91 \mathrm{eV}$ [13] PBE band gap for black-P, 1.50 [13] and $1.59 \mathrm{eV}$ [53] HSE06 band gap for black-P, and $1.98 \mathrm{eV}$ [27] PBE band gap for blue-P.

Before discussing the thermoelectric coefficients of phosphorenes, a few notes on their phononic properties are in order. The calculated results for the vibrational spectra of single layers of blue-P and black-P are depicted along the high symmetry $q$-points in figures 2 (c) and (d). We predict quite similar frequency ranges reflecting the similar bonding characteristics of the two structures as previously reported [27]. The calculated phonon frequencies are free from imaginary frequencies over the whole $q$-space, which ensures the accurate determination of lattice thermal properties of the materials. Phonon dispersions of black-P along $\mathrm{ZZ}$ and $\mathrm{AC}$ directions display important differences. Acoustic modes of black-P have higher group velocities in the AC direction, but the number of modes are higher at lower energies in the $\mathrm{ZZ}$ 

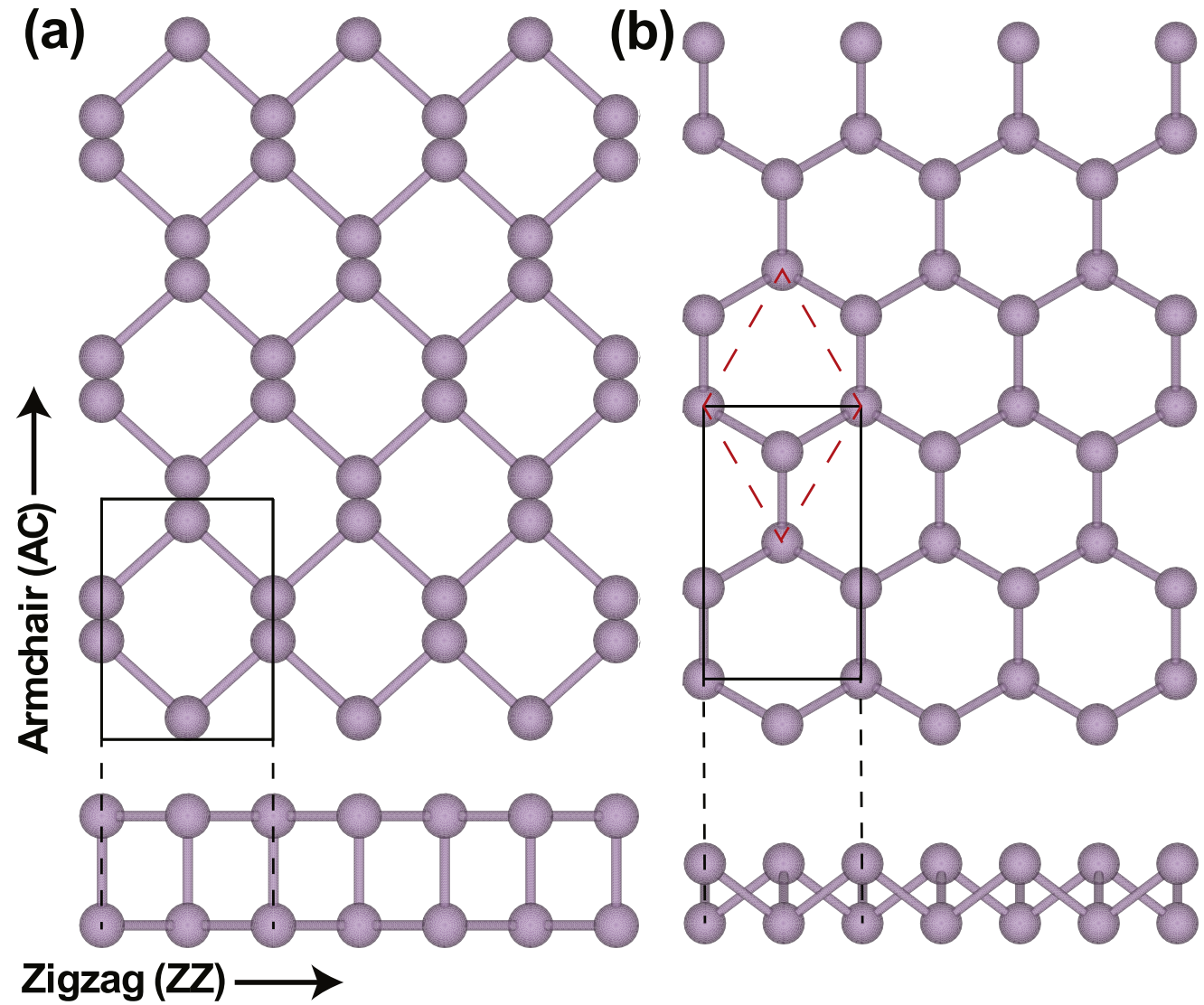

Figure 1. Schematic description of in plane and out of plane views of (a) black-P and (b) blue-P structures. The black tetragonal cells represent the conventional cells used in transport calculations and the red dashed cells represent the corresponding unit cell for blue-P.
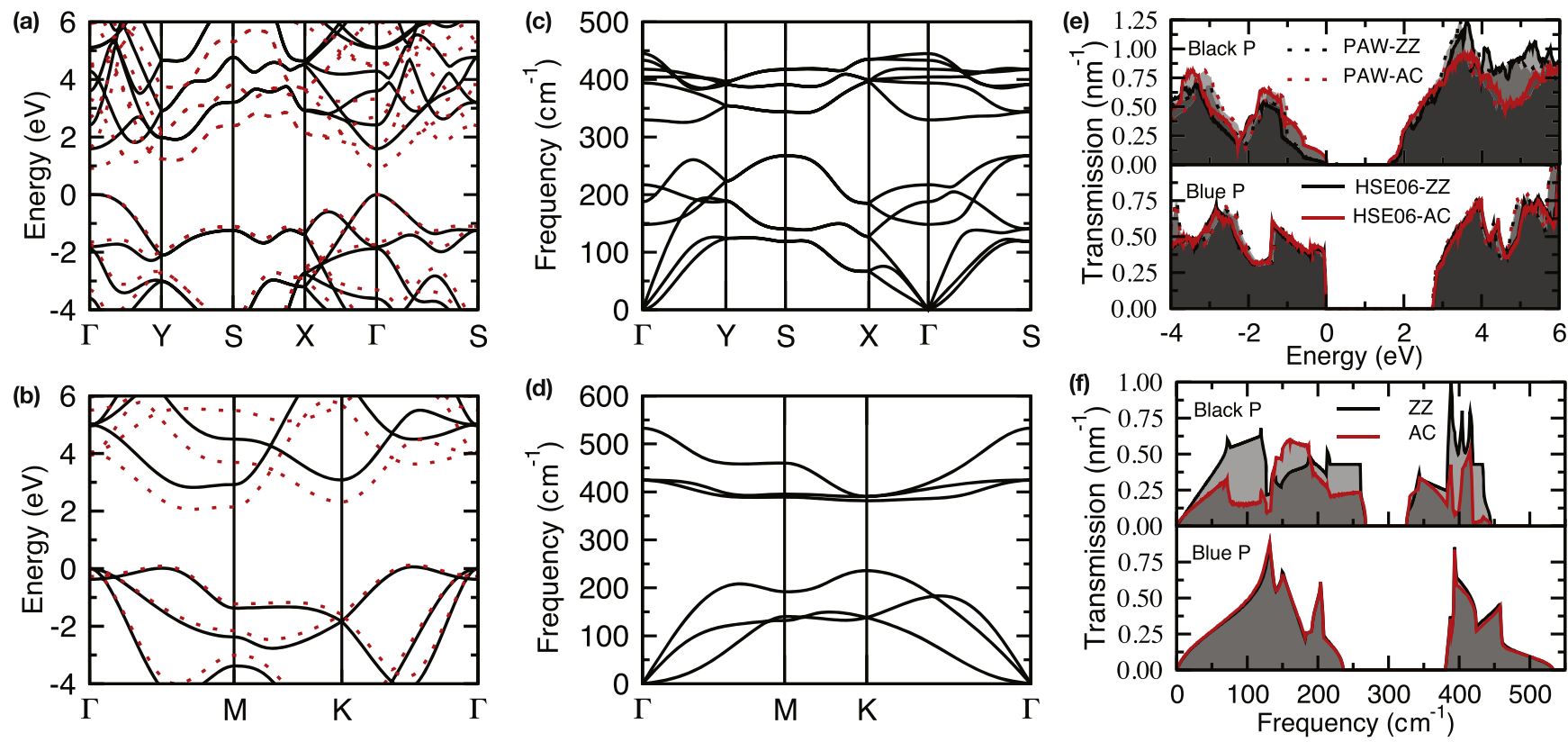

Figure 2. Electronic band structure of (a) black-P and (b) blue-P mono layers calculated using HSE06 (black solid lines) and PBE (red dotted lines) functionals. Vibrational band structure, $\omega(\mathbf{q})$ of (c) black-P and (d) blue-P mono layers. Ballistic electron (e) and phonon (f) transmission spectra of black-P and blue-P mono layers calculated by HSE06 (solid lines) and PBE (dotted lines) functionals. The black and red lines represent the transmission through zigzag (ZZ) and armchair (AC) directions, shown in figure 1, respectively. 
(a) $\times 10^{-4}$

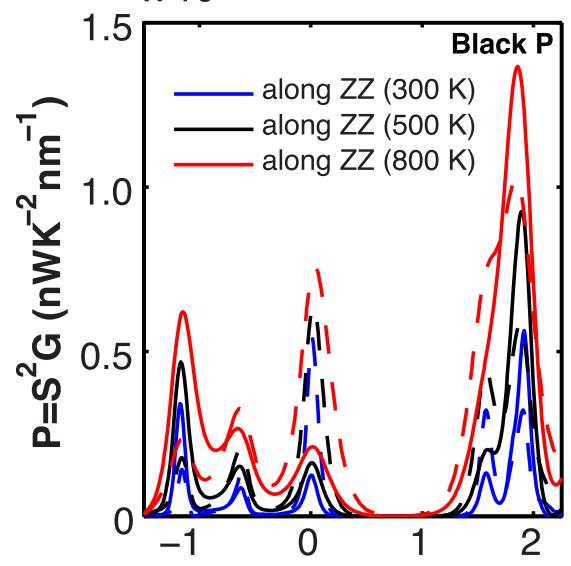

(d) $\times 10^{-4}$

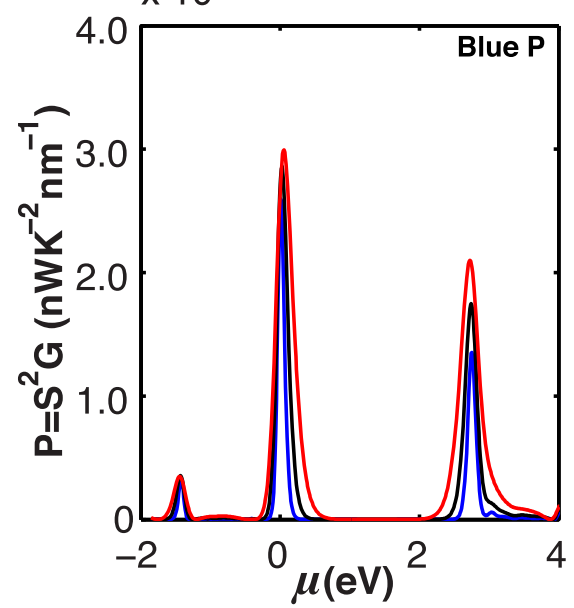

(b)

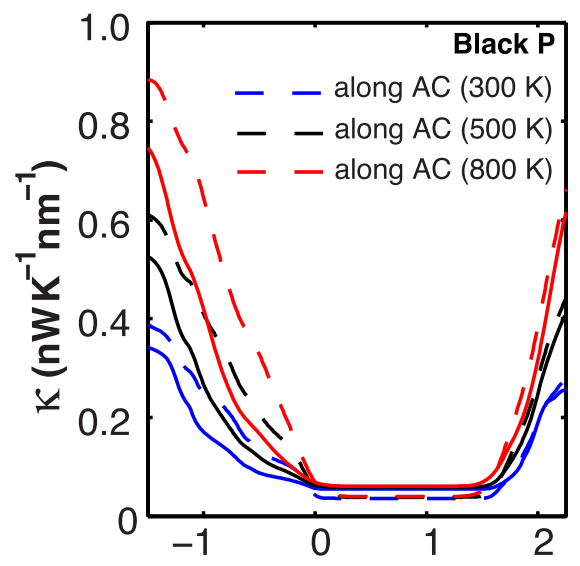

(e)

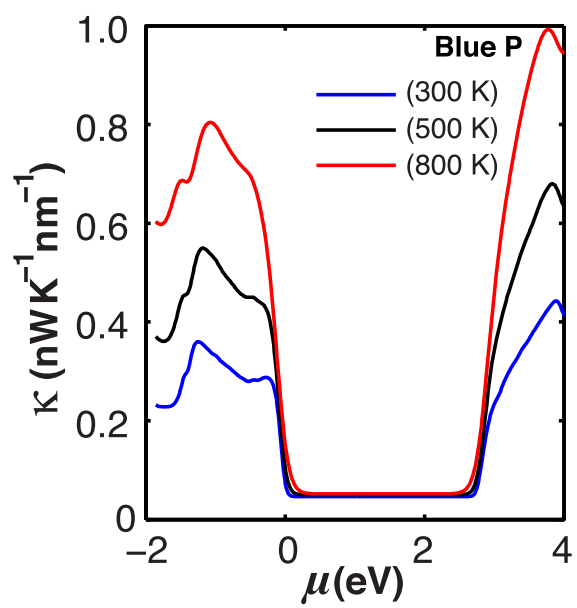

(c)

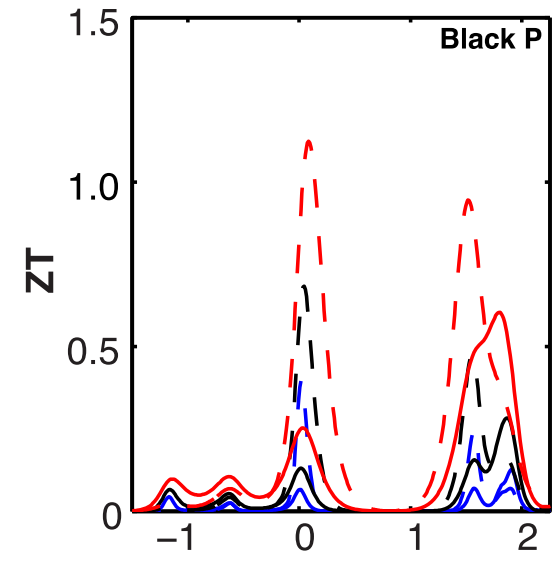

(f)

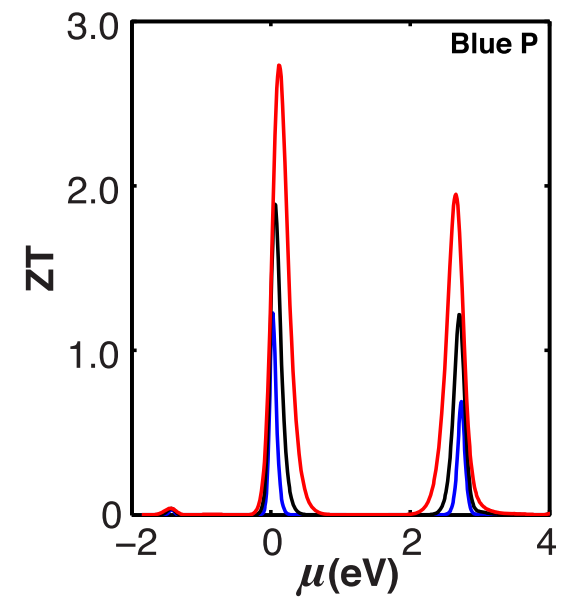

Figure 3. Thermoelectric coefficients of black-P and blue-P structures. The power factor $P=S^{2} G$, (a and d) electronic thermal conductance $\kappa_{\mathrm{el}} / A$, (b and e) and $Z T$ corresponding to the temperatures 300,500 , and $800 \mathrm{~K}$ (c and $\mathrm{f}$ ). The labels $\mathrm{ZZ}$ and $\mathrm{AC}$ represent the armchair and zigzag directions shown in figure 1 .

direction. As a result, phonon transmission is higher in the $\mathrm{ZZ}$ direction for low energy acoustic phonons up to $\sim 150 \mathrm{~cm}^{-1}$. Above $150 \mathrm{~cm}^{-1}$, transmission is higher in the AC direction. Since the low energy phonons are dominant in thermal energy transport, we observe a higher $\kappa_{\mathrm{ph}}$ in the $\mathrm{ZZ}$ direction by a factor of 0.66 , which is close to the values predicted previously with first-principles based solution of the phonon Boltzmann transport equation (0.45 [29] and 0.57 [32]) and non-equilibrium Green's function calculations (0.70 [30]). Both black-P and blue-P have phonon band gaps, starting at similar frequencies (30 meV), blue-P's band gap being larger by a factor of $\sim 2.5(19 \mathrm{meV})$. Such a band gap also has observable effects in thermal transport. At room temperature, black-P has $0.55(0.35) \mathrm{nW} \mathrm{K}^{-1} \mathrm{~nm}^{-1}$ in the $\mathrm{ZZ(AC)}$ direction, while blue-P has $0.45 \mathrm{nW} \mathrm{K}^{-1} \mathrm{~nm}^{-1}$. At $1000 \mathrm{~K}$, these values are increased as $0.61(0.40)$ and $0.52 \mathrm{nW} \mathrm{K}^{-1} \mathrm{~nm}^{-1}$, respectively. Note that the maximum energies of the phonon modes correspond to $640 \mathrm{~K}$ and $780 \mathrm{~K}$ for black-P and blue-P respectively.

Electronic transmission spectra, $\tau_{\text {el }}$ calculated with both HSE06 and PBE functionals are given in figure 2(e) (The band gap values of two calculations are equalized). The electronic band structures of black-P and blue-P are quite different and this difference is reflected in their transmission spectra. In particular, transmission changes abruptly at the valence band edge of blue-P. This is a sign for high thermopower, which depends on the logarithmic derivative of the transmission function. In figure 3 , we plot the power factor $(P)$, thermal conductance $\left(\kappa=\kappa_{\mathrm{el}}+\kappa_{\mathrm{ph}}\right)$ and thermoelectric figure of merit $(Z T)$ for black-P and blue-P as functions of the chemical potentials at three different temperatures, $T=300$, 500 and $800 \mathrm{~K}$. Since the Seebeck coefficient is defined as $S=\left(L_{1} / L_{0}\right) / e T$, it returns extremely high but physically insignificant values inside the band gap. Power factor, $S^{2} G=L_{1}^{2} / L_{0} T^{2}$, yields purposeful results with a more direct connection to $Z T$. In figures 3(a), (d) power factors are shown as functions of chemical potential for black-P and blue-P respectively at different temperatures $(300,500,800 \mathrm{~K})$. Moreover, the maximum $P$ values at the valence and conduction band edges are also reported in table 1 . Indeed, these results are direct consequences of the transmission spectra of both structures. 
Table 1. Calculated power factor values at close to the valence, $P_{V}$ and conduction, $P_{C}$ band edges, in units of $\mathrm{pW} \mathrm{K}^{-2} \mathrm{~nm}^{-1}$ at different temperatures $(300,500,800 \mathrm{~K})$.

\begin{tabular}{|c|c|c|c|c|c|c|}
\hline \multirow[b]{2}{*}{$\mathrm{T}$} & \multicolumn{2}{|c|}{ Black-P ZZ } & \multicolumn{2}{|c|}{ Black-P AC } & \multicolumn{2}{|c|}{ Blue-P } \\
\hline & $P_{V}$ & $P_{C}$ & $P_{V}$ & $P_{C}$ & $P_{V}$ & $P_{C}$ \\
\hline 300 & 0.012 & 0.013 & 0.055 & 0.034 & 0.260 & 0.140 \\
\hline 500 & 0.016 & 0.020 & 0.065 & 0.045 & 0.290 & 0.180 \\
\hline 800 & 0.021 & 0.045 & 0.075 & 0.080 & 0.300 & 0.210 \\
\hline
\end{tabular}

In figures 3(b) and (e) thermal conductance values are plotted as functions of the chemical potential at $T=300,500$ and $800 \mathrm{~K}$. The values inside the band gaps are due to the phonons which are independent of the chemical potential. The relative contributions of electrons and phonons to thermal conductance is an indication of thermoelectric efficiency. If $\kappa$ is dominated by the phononic contribution, most of the heat is transported without participating in electric current. In phosphorenes, $\kappa$ is dominated by electrons unless $\mu$ is inside a band gap, which is an indication of high thermoelectric efficiency.

The resulting $Z T$ values are plotted in figures 3(c) and (f) as functions of $\mu$. The maxima are achieved in the proximity of the valence and conductance band edges. For black-P, $Z T=0.13(0.4)$ is achieved in the $\mathrm{ZZ}(\mathrm{AC})$ direction at room temperature, while $0.6(1.15)$ is possible at $T=800 \mathrm{~K}$. These values are remarkably smaller than those predicted with the solution of semiclassical Boltzmann Transport equation (6.4 [35], and 2.1 [36]). However, our ballistic transport results are in parallel with the first-principles calculations considering the electron-phonon interaction in black-P based on density functional perturbation theory and Wannier interpolation (the maximum room temperature $Z T$ values of $p$ - and $n$-type black-P along armchair direction are around 0.06 and 0.04 respectively) [37]. Conversely, we predict quite high room temperature $Z T$ values as high as $1.25(0.7)$ valence (conduction) electrons of blue-P. Furthermore, significantly high $Z T$ values $(\sim 2.5)$ is possible at elevated temperatures. The maximum $Z T$ figures are plotted for black-P (ZZ and AC directions) and blue-P as functions of temperature in figure 4. One observes an almost linear dependence on temperature for all three plots, which indicates that $Z$ are almost independent of temperature for phosphorenes.

In summary, our first-principles investigation explicitly states that previous calculations [28, 35, 36], treating the electron-phonon interaction with constant relaxation time approximation and estimating the electrical conductivity with deformation potential approximation, estimate the thermoelectric efficiency of black-P to be beyond their ballistic values. Thermoelectric efficiency sufficient for technological applications might be only possible at high temperatures for black-P. On the other hand, our ballistic quantum transport investigation conspicuously indicates the much better thermoelectric performance of blue-P. We attribute the rather higher maximum $Z T$ values of blue-P to its high power factor resulting from the large slope in the transmission function

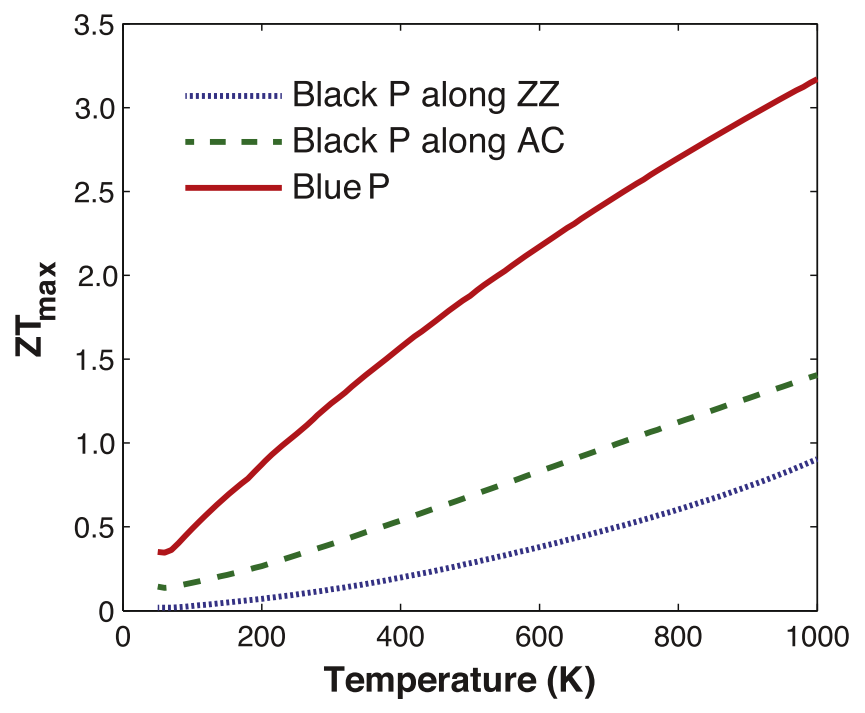

Figure 4. Maximum $Z T$ as a function of temperatures corresponding to black-P and blue-P structures. The labels $\mathrm{ZZ}$ and $\mathrm{AC}$ represent the armchair and zigzag directions shown in figure 1.

around band edges, in agreement with the Mahan-Sofo criteria [54].

\section{Acknowledgments}

We would like to thank the ULAKBIM High Performance and Grid Computing Center for a sufficient time allocation for our projects. C S acknowledges the support from Scientific and Technological Research Council of Turkey (TUBITAK113F096), Anadolu University (BAP-140F335), and Turkish Academy of Sciences (TUBA-GEBIP). H S acknowledges support from Scientific and Technological Research Council of Turkey (BIDEB-113C032) and Bilim Akademisi-the Science Academy, Turkey under the BAGEP program.

\section{References}

[1] Miro P, Audiffred M and Heine T 2014 Chem. Soc. Rev. 43 6537-54

[2] Zhi C, Bando Y, Tang C, Kuwahara H and Golberg D 2009 Adv. Mater. 21 2889-93

[3] Zeng H, Zhi C, Zhang Z, Wei X, Wang X, Guo W, Bando Y and Golberg D 2010 Nano Letters 10 5049-55

[4] Nag A, Raidongia K, Hembram K P S S, Datta R, Waghmare U V and Rao C N R 2010 ACS Nano 4 1539-44

[5] Aufray B, Kara A, Vizzini S, Oughaddou H, Léandri C, Ealet B and Le Lay G 2010 Appl. Phys. Lett. 96183102

[6] Feng B, Ding Z, Meng S, Yao Y, He X, Cheng P, Chen L and Wu K 2012 Nano Letters 12 3507-11

[7] Vogt P, De Padova P, Quaresima C, Avila J, Frantzeskakis E, Asensio M C, Resta A, Ealet B and Le Lay G 2012 Phys. Rev. Lett. 108155501

[8] Meng L et al 2013 Nano Letters 13 685-90

[9] Wang Q H, Kalantar-Zadeh K, Kis A, Coleman J N and Strano M S 2012 Nat Nano 7 699-712 
[10] Radisavljevic B, Radenovic A, Brivio J, Giacometti V and Kis A 2011 Nat Nano 6 147-50

[11] Chhowalla M, Liu Z and Zhang H 2015 Chem. Soc. Rev. 44 2584-6

[12] Chia X, Eng A Y S, Ambrosi A, Tan S M and Pumera M 2015 Chem. Rev. 11511941

[13] Qiao J, Kong X, Hu Z X, Yang F and Ji W 2014 Nat. Commun. 54475

[14] Li L, Yu Y, Ye G J, Ge Q, Ou X, Wu H, Feng D, Chen X H and Zhang Y 2014 Nat. Nano 9 372-7

[15] Wang X, Jones A M, Seyler K L, Tran V, Jia Y, Zhao H, Wang H, Yang L, Xu X and Xia F 2015 Nat. Nano 10 517-21

[16] Tran V, Soklaski R, Liang Y and Yang L 2014 Phys. Rev. B 89 235319

[17] Xiao J, Long M, Zhang X, Ouyang J, Xu H and Gao Y 2015 Sci. Rep. 59961

[18] Li X B, Guo P, Cao T F, Liu H, Lau W M and Liu L M 2015 Sci. Rep. 510848

[19] Kim J S, Liu Y, Zhu W, Kim S, Wu D, Tao L, Dodabalapur A, Lai K and Akinwande D 2015 Sci. Rep. 58989

[20] Liu H, Neal A T, Zhu Z, Luo Z, Xu X, Tománek D and Ye P D 2014 ACS Nano 8 4033-41

[21] Fei R and Yang L 2014 Nano Letters 14 2884-9

[22] Buscema M, Groenendijk D J, Blanter S I, Steele G A, van der Zant H S J and Castellanos-Gomez A 2014 Nano Letters 14 3347-52

[23] Çakır D, Sahin H and Peeters F M 2014 Phys. Rev. B 90205421

[24] Li W, Yang Y, Zhang G and Zhang Y W 2015 Nano Letters 15 $1691-7$

[25] Wu M, Fu H, Zhou L, Yao K and Zeng X C 2015 Nano Letters 15 3557-62

[26] Guan J, Zhu Z and Tománek D 2014 Phys. Rev. Lett. 113 046804

[27] Zhu Z and Tománek D 2014 Phys. Rev. Lett. 112176802

[28] Fei R, Faghaninia A, Soklaski R, Yan J A, Lo C and Yang L 2014 Nano Letters 14 6393-9

[29] Qin G, Yan Q B, Qin Z, Yue S Y, Hu M and Su G 2015 Phys. Chem. Chem. Phys. 17 4854-8
[30] Ong Z Y, Cai Y, Zhang G and Zhang Y W 2014 J. Phys. Chem. C 118 25272-7

[31] Zhu L, Zhang G and Li B 2014 Phys. Rev. B 90214302

[32] Liu T H and Chang C C 2015 Nanoscale 7 10648-54

[33] Jain A and McGaughey A J H 2015 Sci. Rep. 58501

[34] Balandin A A 2011 Nat. Mater 10 569-81

[35] Zhang J, Liu H J, Cheng L, Wei J, Liang J H, Fan D D, Shi J, Tang X F and Zhang Q J 2014 Sci. Rep. 46452

[36] Lv H Y, Lu W J, Shao D F and Sun Y P 2014 Phys. Rev. B 90 085433

[37] Liao B, Zhou J, Qiu B, Dresselhaus M S and Chen G 2015 Phys. Rev. B 91235419

[38] Kresse G and Hafner J 1993 Phys. Rev. B 47 558-61

[39] Wu X, Vanderbilt D and Hamann D R 2005 Phys. Rev. B 72 035105

[40] Kohn W and Sham L J 1965 Phys. Rev. 140 A1133-8

[41] Hohenberg P and Kohn W 1964 Phys. Rev. 136 B864-71

[42] Martin R M 2004 Electronic Structure (Cambridge, England: Cambridge University Press)

[43] Blöchl P E 1994 Phys. Rev. B 50 17953-79

[44] Kresse G and Joubert D 1999 Phys. Rev. B 59 1758-75

[45] Perdew J P, Burke K and Ernzerhof M 1996 Phys. Rev. Lett. 77 $3865-8$

[46] Krukau A V, Vydrov O A, Izmaylov A F and Scuseria G E 2006 J. Chem. Phys. 125224106

[47] Becke A D 1993 J. Chem. Phys. 98 1372-7

[48] Togo A, Oba F and Tanaka I 2008 Phys. Rev. B 78134106

[49] Baroni S, de Gironcoli S, Dal Corso A and Giannozzi P 2001 Rev. Mod. Phys. 73 515-62

[50] Rego L G C and Kirczenow G 1998 Phys. Rev. Lett. 81 232-5

[51] Esfarjani K, Zebarjadi M and Kawazoe Y 2006 Phys. Rev. B $\mathbf{7 3} 085406$

[52] Aierken Y, Çakır D, Sevik C and Peeters F M 2015 Phys. Rev. B 92081408

[53] Çakır D, Sevik C and Peeters F M 2015 Phys. Rev. B 92 165406

[54] Mahan G D and Sofo J O 1996 Proc. Nat. Acad. Sci. 93 7436-9 